Classification

Physics Abstracts

$05.40-68.70-02.50$

\title{
Path integral approach to birth-death processes on a lattice
}

\author{
L. Peliti \\ Dipartimento di Fisica, Universita « La Sapienza », Piazzale Aldo Moro 2, I-00185 Roma \\ and Gruppo Nazionale di Struttura della Materia, Unità di Roma, Roma, Italy
}

(Reçu le 8 mars 1985, accepté le 22 mai 1985)

\begin{abstract}
Résumé. - On donne une formulation par intégrales de chemin du formalisme de Fock pour objets classiques, premièrement introduit par Doi, et on l'applique à des processus généraux de naissance et mort sur réseau. L'introduction de variables auxiliaires permet de donner une forme Markovienne aux lois d'évolution des chemins aléatoires avec mémoire et des processus irréversibles d'agrégation. Les théories des champs existantes pour ces processus sont obtenues dans la limite continue. On discute brièvement des implications de cette méthode pour leur comportement asymptotique.
\end{abstract}

\begin{abstract}
The Fock space formalism for classical objects first introduced by Doi is cast in a path integral form and applied to general birth-death processes on a lattice. The introduction of suitable auxiliary variables allows one to formulate random walks with memory and irreversible aggregation processes in a Markovian way, which is treatable in this formalism. Existing field theories of such processes are recovered in the continuum limit. Implications of the method for their asymptotic behaviour are briefly discussed.
\end{abstract}

\section{Introduction.}

A certain number of models of irreversible aggregation processes, which lead to the formation of objects possessing some sort of scale invariance, have been introduced in the literature since the proposal of Witten and Sander [1]. One of the main reasons of interest appears to be the hope of understanding the origin of the scale invariance of many natural objects by widening the scope of methods, like the renormalization group, which have proven their validity in the description of scale invariance in critical phenomena.

This program does not yet appear to have reached its target. While the number of model increases, most investigations still essentially rely on numerical simulation $[2,3]$. Sometimes arguments which link the evolution equations which define the model to the geometrical properties of the outcoming aggregate have appeared [4-8]. Methods which are strictly related to the renormalization group have met with some success in the particular case of growing linear aggregates, which may be produced by processes described as random walks with memory [9-13]. In this case also Flory-like arguments have found some applicability $[14,15]$. One of the reasons of success has been the introduction of a field theory describing the process, which has a renormalizable perturbation theory around some upper critical dimension $D_{c}$. For the case of random walks with memory such field theories have been introduced on a heuristic basis $[12,13,16]$. On the other hand, field theories describing some form of aggregation processes or chemical reactions with diffusion have been from time to time introduced and discussed in the literature [17-21]. The treatment has been extended to account for memory effects [22].

We wish here to bring together these field theories and show how one can systematically take memory effects into account, on the basis of the Fock space formalism for classical objects first introduced by Doi [23], and later reformulated by Rose [24] and Grassberger and Scheunert [25]. The method bears strong similarity to the Martin-Siggia-Rose formalism for classical evolution [26], which has been cast into a path integral form by De Dominicis [27] and Janssen [28]. It is also related to the Poisson representation of birth-death processes introduced by Gardiner and Chaturvedi [29], on which basis Janssen [20] has been able to give a path integral formulation of some chemical reaction models with diffusion.

Although such methods have from time to time appeared in literature, we think it worthwhile to explain them in a slightly different way, which closely 
follows the treatment of Bargmann-Fock path integrals in standard texts on Quantum Field Theory (see e.g. [30]). One can thus most clearly derive the exact definition of the path integral and its properties. One can stress the flexibility of the formalism by a derivation which does not unnecessarily reduce the symmetry existent among the various fields which are introduced. Some cases which allow for an exact treatment are thus most easily spotted and handled.

It is also convenient to treat in some detail the trick which allows one to transform an aggregation process with memory into a process obeying a Markovian evolution equation via the introduction of "bookkeeping variables». This trick has been suggested by Grassberger [31] and has wide applicability. We show how it leads to a sound derivation of field theories of aggregation processes with memory which have appeared in the literature.

Once the field theories have been defined, a renormalization procedure may be set up to discuss the asymptotic behaviour of the model. This procedure may succeed or fail depending on the availability of a small parameter (like the $\varepsilon=4-D$ of static critical phenomena) which allows one to bring the fixed points of the renormalization group within the range of applicability of perturbation theory. We shall not pursue in detail the renormalization group treatment, which is rather standard once the correct field theory is identified. We shall however mention the reasons leading to difficulties in applying this approach to some popular aggregation models.

We review in section 2 the derivation of the path integral formalism for classical, zero-dimensional, birth-death processes. Some simple examples and the perturbation theory for such processes are discussed in section 3. The treatment of birth-death processes without memory on a lattice is sketched in section 4. In section 5 the trick of the " bookkeeping variables " for the treatment of processes with memory is introduced and some inferences on the asymptotic behaviour of a few interesting processes are drawn. While section 6 contains a short conclusion, the discussion of a technical detail is dealt with in an appendix.

\section{Path integrals for birth-death processes.}

We start by considering zero-dimensional birth-death processes whose microscopic states are completely described by the number $n$ of particles present at any time $t$.

Its macroscopic state is therefore described by the probability $\phi_{n}(t)$ of having exactly $n$ particles at time $t$. This probability evolves according to a master equation of the form :

$$
\frac{\mathrm{d} \phi_{n}(t)}{\mathrm{d} t}=\sum_{n\left(\neq n^{\prime}\right)}\left[w\left(n^{\prime} \rightarrow n\right) \phi_{n^{\prime}}(t)-w\left(n \rightarrow n^{\prime}\right) \phi_{n}(t)\right] .
$$

The factors $w\left(n^{\prime} \rightarrow n\right)$ are the transition rates from the state with $n^{\prime}$ particles to the state with $n$ particles. In a process without memory (as we shall suppose throughout this section) they can only depend on $n^{\prime}, n$ (and possibly on time).

The macroscopic state identified by $\left\{\phi_{n}\right\}$ may be considered as an element of a real vector space $H$ formed by linear combinations of the base states $|n\rangle$ corresponding to states with exactly $n$ particles :

$$
|\phi\rangle=\sum_{n} \phi_{n}|n\rangle \text {. }
$$

The space may be given a Hilbert structure by introducing the scalar product

$$
\langle n \mid m\rangle=n ! \delta_{n m} .
$$

This is the "exclusive " scalar product in the terminology of Grassberger and Scheunert [25]. These authors also introduce a different scalar product, which they name "inclusive". This product leads to some simplifications in the formalism, which we shall discuss later. We refer to the appendix for a brief discussion of the properties of the inclusive scalar product.

We can thus introduce the annihilation operator $a$ satisfying

$$
a|n\rangle=n|n-1\rangle,
$$

and the creation operator $\pi$ defined by

$$
\pi|n\rangle=|n+1\rangle \text {. }
$$

With the scalar product (2.3) one may easily check that $\pi$ is the Hermitian conjugate of $a$. The operators $a, \pi$ have the usual commutation relation

$$
[a, \pi]=1 \text {. }
$$

A look at equations (2.4)-(2.5) suggests to represent $\mathcal{H}$ as a Hilbert space of (real) analytic functions of the variable $z$ by means of the correspondence

$$
|\phi\rangle=\sum_{n} \phi_{n}|n\rangle \leftrightarrow \phi(z)=\sum_{n} \phi_{n} z^{n}
$$

with real Taylor coefficients $\phi_{n}$. We shall however allow $z$ to take on complex values.

The function $\phi(z)$ associated with the state $|\phi\rangle$ is of course nothing else as the generating function, well known in the theory of birth-death processes $[32,33]$. We are thus providing the space of generating functions with a Hilbert space structure.

Let us remark that the scalar product between two arbitrary vectors $|\phi\rangle,|\psi\rangle$ is given by

$$
\langle\phi \mid \psi\rangle=\sum_{n} \frac{1}{n !}\langle\phi \mid n\rangle\langle n \mid \psi\rangle=\sum_{n} n ! \phi_{n} \psi_{n} .
$$


By the use of the identity ([33], p. 280) :

$$
n ! \delta_{n m}=\int \mathrm{d} z z^{n}\left(-\frac{\mathrm{d}}{\mathrm{d} z}\right)^{m} \delta(z),
$$

which one can easily check by integrating by parts, we express $(2.8)$ in terms of the generating functions $\phi(z), \psi(z)$ as follows :

$$
\langle\phi \mid \psi\rangle=\int \mathrm{d} z \phi(z) \psi\left(-\frac{\mathrm{d}}{\mathrm{d} z}\right) \delta(z) .
$$

The use of the usual integral representation of the delta function allows finally to express the scalar product (2.8) as an integral :

$$
\langle\phi \mid \psi\rangle=\int \frac{\mathrm{d} z \mathrm{~d} z^{\prime}}{2 \pi} \phi(z) \psi\left(i z^{\prime}\right) \mathrm{e}^{-i z z^{\prime}} .
$$

The integral over $z^{\prime}$ runs over the whole real axis; that over $z$ over any path which passes through zero. We shall always understand that they are both taken over the whole real axis.

The evolution equation (2.1) may be symbolically written as follows :

$$
\frac{\mathrm{d}|\phi(t)\rangle}{\mathrm{d} t}=L|\phi(t)\rangle
$$

where we have introduced the evolution operator $L$, also called the "Liouvillian ". The formal solution of equation (2.12) is

$$
|\phi(t)\rangle=\exp (t L)|\phi(0)\rangle .
$$

Our aim is to express the evolution operator $U_{t}=\exp (t L)$ as a functional integral. To do this let us express as an integral the effect of any operator $A$ on a state $|\phi\rangle$ belonging to $\mathscr{H}$. Given the matrix elements $A_{n m}$ of $A$ we associate to it the kernel :

$$
A(z, \zeta)=\sum_{m n} \frac{z^{m}}{m !} A_{m n} \frac{\zeta^{n}}{n !} .
$$

We then have the following correspondence :

$$
|\psi\rangle=A|\phi\rangle \leftrightarrow \psi(z)=\int \frac{\mathrm{d} \zeta \mathrm{d} \zeta^{\prime}}{2 \pi} A(z, \zeta) \phi\left(i \zeta^{\prime}\right) \mathrm{e}^{-i \zeta \zeta^{\prime}} .
$$

The kernel of a product $A B$ of operators is given by :

$$
A B(z, \zeta)=\int \frac{\mathrm{d} \eta \mathrm{d} \eta^{\prime}}{2 \pi} A(z, \eta) B\left(i \eta^{\prime}, \zeta\right) \mathrm{e}^{-i \eta \eta^{\prime}}
$$

The kernel of any operator $A$ is easily computable if $A$ is first expressed as a normal product, i.e. as a polynomial in $a, \pi$ with all creation operators on the left of all destruction operators. It is always possible to express any operator in such a form. Given the normal product expression of $\boldsymbol{A}$ :

$$
A=\sum_{m n} \mathcal{A}_{m n} \pi^{m} a^{n},
$$

the normal kernel $\mathcal{A}(z, \zeta)$ of $A$ is simply defined as

$$
\mathcal{A}(z, \zeta)=\sum_{m n} \mathcal{A}_{m n} z^{m} \zeta^{n},
$$

where $z$ takes the place of $\pi$ and $\zeta$ of $a$; and the kernel $A(z, \zeta)$ of $A$ is given by

$$
A(z, \zeta)=\mathrm{e}^{z \zeta} \mathcal{A}(z, \zeta) .
$$

The derivation of equations (2.16)-(2.19) is the same as in quantum mechanics and may be found, e.g., in reference [30].

To express $U_{t}=\exp (t L)$ we apply the Trotter formula :

$$
U_{t}=\exp (t L)=\lim _{N \rightarrow \infty}\left(1+\frac{t}{N} L\right)^{N} .
$$

The kernel of each factor $(1+(t / N) L)$ is expressed in terms of the normal kernel $\mathfrak{L}(z, \zeta)$ of $L$ as follows :

$$
\left(1+\frac{t}{N} L\right)(z, \zeta)=\mathrm{e}^{z \zeta}\left[1+\frac{t}{N} \mathfrak{L}(z, \zeta)\right]
$$

We have therefore :

$$
\begin{gathered}
U_{t}(z, \zeta)=\lim _{N \rightarrow \infty} \int_{l=1}^{N-1}\left(\frac{\mathrm{d} \eta_{l} \mathrm{~d} \eta_{l}^{\prime}}{2 \pi} \mathrm{e}^{-i \eta^{\prime} \eta_{l}}\right) \\
\prod_{l=1}^{N}\left\{\left[1+\frac{t}{N} \mathfrak{L}\left(i \eta_{l}^{\prime}, \eta_{l-1}\right)\right] \mathrm{e}^{i \eta^{\prime} \eta_{l-1}}\right\} .
\end{gathered}
$$

The boundary conditions are given by :

$$
i \eta_{N}^{\prime}=z ; \quad \eta_{0}=\zeta
$$

Equation (2.21) may be written as follows :

$$
\begin{gathered}
U_{t}(z, \zeta)=\lim _{N \rightarrow \infty} \int_{l=1}^{N-1} \frac{\mathrm{d} \eta_{l} \mathrm{~d} \eta_{l}^{\prime}}{2 \pi} \\
\exp \left\{\sum_{l=1}^{N-1}\left[-i \eta_{l}^{\prime}\left(\eta_{l}-\eta_{l-1}\right)+\frac{t}{N} \mathfrak{L}\left(i \eta_{l}^{\prime}, \eta_{l-1}\right)\right]+\frac{t}{N} \mathfrak{L}\left(z, \eta_{N-1}\right)+z \eta_{N-1}\right\} .
\end{gathered}
$$

The term under summation in the exponent of equation (2.23) may be formally expressed as an integral. We 
obtain therefore the path integral expression of $U_{t}$ :

$$
U_{t}(z, \zeta)=\int \mathfrak{D} \eta \mathfrak{D} \eta^{\prime} \exp \left\{-\int_{0}^{t} \mathrm{~d} t^{\prime}\left[i \eta^{\prime}\left(t^{\prime}\right) \dot{\eta}\left(t^{\prime}\right)-\mathfrak{L}\left(i \eta^{\prime}\left(t^{\prime}\right), \eta\left(t^{\prime}\right)\right)\right]+z \eta(t)\right\}
$$

where the dot represents the time derivative, and where the following boundary conditions are understood :

$$
\eta(0)=\zeta ; \quad i \eta^{\prime}(t)=z .
$$

The factors $(2 \pi)$ are incorporated in the functional integration measure.

Let us express the expectation value of the operator $A$ in the state $|\phi\rangle=\sum_{n} \phi_{n}|n\rangle$. Let us remark that operators corresponding to observables are diagonal in the basis of states $|n\rangle$ with definite particle number, whereas we may say that operators which are not diagonal in this basis correspond to processes. The expectation value of an observable is given by

$$
\langle A\rangle_{\phi}=\sum_{n} A(n) \phi_{n}
$$

where $A(n)$ is the value the operator $A$ assumes in the state $|n\rangle$. We have

$\langle A\rangle_{\phi}=\sum_{n m} \frac{1}{n !} \frac{1}{m !}\langle n|A| m\rangle\langle m \mid \phi\rangle=\langle|A| \phi\rangle$,

where we have introduced the "standard bra"

$$
\langle|=\sum_{n} \frac{1}{n !}\langle n| \text {. }
$$

We shall define analogously expectation values of operators which are not observables. It is worthwhile to remark that since

$$
\langle|=\langle 0| \mathrm{e}^{a},
$$

one has for any integer $k$

$$
<\mid \pi^{k}=<1 \text {. }
$$

This allows us to write down quite simply the expectation value of any operator given in its normal form. In particular, since observables must have the expression

$$
A=\sum_{k} \mathfrak{A}_{k k} \pi^{k} a^{k},
$$

we have

$$
\langle A\rangle_{\phi}=\langle|A| \phi\rangle=\sum_{k} \mathcal{A}_{k k}\left\langle\left|a^{k}\right| \phi\right\rangle .
$$

Going now to the generating function representation, taking into account equation (2.29) and the fact that $|0\rangle$ is represented by the function 1 , we obtain

$$
\langle A\rangle_{\phi}=\int \frac{\mathrm{d} z \mathrm{~d} z^{\prime}}{2 \pi} \int \frac{\mathrm{d} \zeta \mathrm{d} \zeta^{\prime}}{2 \pi} \mathrm{e}^{z-i z^{\prime} z} A\left(i z^{\prime}, \zeta\right) \mathrm{e}^{-i \zeta^{\prime} \zeta} \phi\left(i \zeta^{\prime}\right),
$$

which simplifies for observables as follows :

$$
\langle A\rangle_{\phi}=\int \frac{\mathrm{d} z \mathrm{~d} z^{\prime}}{2 \pi} \mathrm{e}^{z} \sum_{k} \mathcal{A}_{k k} z^{k} \phi\left(i z^{\prime}\right),
$$

and for a general operator having the normal form (2.18) :

$$
\langle A\rangle_{\phi}=\int \frac{\mathrm{d} z \mathrm{~d} z^{\prime}}{2 \pi} \mathrm{e}^{z} \sum_{j k} A_{j k} z^{k} \phi\left(i z^{\prime}\right) .
$$

These expressions are slightly simpler in the inclusive scalar product formalism (see the appendix).

\section{Examples and perturbation theory.}

The simplest process one can consider is exponential decay, defined by the transition rate

$$
w\left(n \rightarrow n^{\prime}\right)=w n \delta_{n, n^{\prime}+1} .
$$

The Liouvillian is therefore given by

$$
L=w(a-\pi a),
$$

and the kernel of the evolution operator $U_{t}$ by

$U_{t}(z, \zeta)=\int \mathfrak{D} \eta \mathfrak{D} \eta^{\prime} \times$

$\times \exp \left\{-\int_{0}^{t} \mathrm{~d} t^{\prime}\left[i \eta^{\prime} \dot{\eta}+w\left(i \eta^{\prime}-1\right) \eta\right]+z \eta(t)\right\}$.

This integral may be simplified by shifting the integration variable as follows :

$$
i \eta^{\prime} \rightarrow i \hat{\eta}=i \eta^{\prime}-1 \text {. }
$$

This shift is automatic in the inclusive scalar product formalism. The fact that the simplest process thus corresponds to a bilinear Lagrangian is one of the main advantages of this formalism. One has therefore :

$$
\begin{aligned}
& U_{t}(z, \zeta)=\int \mathfrak{D} \eta \mathfrak{D} \hat{\eta} \times \\
& \times \exp \left\{-\int_{0}^{t} \mathrm{~d} t^{\prime}[i \hat{\eta} \dot{\eta}+w i \hat{\eta} \eta]+i \hat{\eta}(t) \eta(t)+\zeta\right\},
\end{aligned}
$$

where account has been taken of the boundary con- 
dition

$$
i \hat{\eta}(t)=z-1 .
$$

To check the result, observe that the integration over $\hat{\eta}$ for $0<t^{\prime}<t$ enforces the constraint

$$
\frac{\mathrm{d} \eta\left(t^{\prime}\right)}{\mathrm{d} t^{\prime}}=-w \eta\left(t^{\prime}\right) .
$$

We have therefore

$$
\eta(t)=\eta(0) \mathrm{e}^{-w t}=\zeta \mathrm{e}^{-w t},
$$

which gives

$$
U_{t}(z, \zeta)=\exp \left[(z-1) \zeta \mathrm{e}^{-w t}+\zeta\right] .
$$

It is easy to check that $\Phi_{t}(z)$, given by

$$
\begin{aligned}
\Phi_{t}(z) & =\int \frac{\mathrm{d} \zeta \mathrm{d} \zeta^{\prime}}{2 \pi} \mathrm{e}^{-i \zeta \zeta^{\prime}} U_{t}(z, \zeta) \Phi_{0}\left(i \zeta^{\prime}\right) \\
& =\Phi_{0}\left((z-1) \mathrm{e}^{-w t}+1\right),
\end{aligned}
$$

does indeed represent the state $\left|\Phi_{t}\right\rangle$ which evolves from $\left|\Phi_{0}\right\rangle$ at time $t=0$ according to the master equation

$$
\frac{\partial\left|\Phi_{t}\right\rangle}{\partial t}=L\left|\Phi_{t}\right\rangle
$$

where $L$ is given by equation (3.2).

The simplest « interacting » processes are branching processes, in which each particle acts independently from any other. As observed by Grassberger and Scheunert [25] this implies that the transition rates $w\left(n \rightarrow n^{\prime}\right)$ are proportional to $n$ and therefore that the Liouvillian $L$ is linear in the destruction operator $a$. Given the transition rates

$$
w\left(n \rightarrow n^{\prime}\right)=\sum_{m \neq 1} \lambda_{m} n \delta_{n, n^{\prime}-m+1},
$$

the Liouvillian has the expression

$$
L=\sum_{m \neq 1} \lambda_{m}\left(\pi^{m}-\pi\right) a .
$$

Introducing the notation

$$
\lambda_{1}=-\sum_{m \neq 1} \lambda_{m},
$$

the Liouvillian assumes the more compact form

$$
L=\sum_{m} \lambda_{m} \pi^{m} a .
$$

The kernel of $U_{t}$ is therefore given by

$$
\begin{aligned}
U_{t}(z, \zeta) & =\int \mathfrak{D} \eta \mathfrak{D} \eta^{\prime} \times \\
& \times \exp \left\{-\int_{0}^{t} \mathrm{~d} t^{\prime}\left[i \eta^{\prime} \dot{\eta}-\sum_{m} \lambda_{m}\left(i \eta^{\prime}\right)^{m} \eta\right]+z \eta(t)\right\} .
\end{aligned}
$$

The functional integral may be evaluated exactly. One first integrates by parts the first term in the exponential obtaining

$$
\begin{aligned}
& U_{t}(z, \zeta)=\int \mathfrak{D} \eta \mathfrak{D} \eta^{\prime} \times \\
& \times \exp \left\{-\int_{0}^{t} \mathrm{~d} t^{\prime}\left[-i \eta \dot{\eta}^{\prime}+i \sum_{m} \lambda_{m} i \eta\left(i \eta^{\prime}\right)^{m}\right]+i \eta^{\prime}(0) \zeta\right\} .
\end{aligned}
$$

Integration over $\eta$ then enforces the constraint

$$
\frac{\mathrm{d}\left(i \eta^{\prime}\right)}{\mathrm{d} t}=-\sum_{m} \lambda_{m}\left(i \eta^{\prime}\right)^{m} .
$$

By changing $t$ into $t-t^{\prime}$ one observes that $i \eta^{\prime}(0)$ is the solution of the differential equation

$$
\frac{\mathrm{d} y}{\mathrm{~d} t^{\prime}}=\sum_{m} \lambda_{m} y^{m}\left(t^{\prime}\right),
$$

satisfying $y(0)=z$, evaluated at $t^{\prime}=t$. Let us call $f(z, t)$ this solution. The kernel $U_{t}(z, \zeta)$ then has the following expression :

$$
U_{t}(z, \zeta)=\exp [\zeta f(z, t)] .
$$

The implies that the generating function $\Phi_{t}(z)$ of the state evolved from the initial state identified by $\Phi_{0}(z)$ at time $t=0$ is given by :

$$
\Phi_{t}(z)=\Phi_{0}(f(z, t)),
$$

which is the well known expression for branched processes (see e.g. Ref. [32], §4, p. 163). By setting $\lambda_{0}=-\lambda_{1}=\lambda, \lambda_{m}=0(m>1)$ we recover, as we should, the previous result. When particles do not act independently, the functional integral can no more be evaluated explicitly in closed form. One must then resort to a perturbation expansion. The simplest such case is Schlögl's first model [34] which is the stochastic version of the Malthus-Verhurlst model for the evolution of populations. Each particle gives rise to another particle with a rate $\lambda$ and disappears with a rate $\mu+v(n-1)$ where $n$ is the overall number of particles. The Liouvillian is therefore :

$$
L=\lambda(\pi-1) \pi a-(\pi-1)(\mu+v \pi a) a,
$$

and the evolution kernel has the expression :

$$
U_{t}(z, \zeta)=\int \mathfrak{D} \eta \mathfrak{D} \eta^{\prime} \exp \left\{-\int_{0}^{t} \mathrm{~d} t^{\prime}\left[i \eta^{\prime} \dot{\eta}-\lambda\left(i \eta^{\prime}-1\right) i \eta^{\prime} \eta+\left(i \eta^{\prime}-1\right)\left(\mu+v i \eta^{\prime} \eta\right)+z \eta(t)\right]\right\} .
$$


The argument of the exponent may be simplified by performing the shift (3.4) or, equivalently, by use of the inclusive scalar product. One thus obtains

$$
U_{t}(z, \zeta)=\int \mathfrak{D} \eta \mathfrak{D} \hat{\eta} \exp \{-S[i \hat{\eta}, \eta]+i \hat{\eta}(t) \eta(t)+\zeta\},
$$

where the boundary conditions $(2.25)$ are understood, and where the action $S$ is given by :

$$
S=S_{0}+S_{1} \text {. }
$$

The free action $S_{0}$ is given by :

$$
S_{0}=\int_{0}^{t} \mathrm{~d} t^{\prime} \mathcal{H}_{0}\left(i \hat{\eta}\left(t^{\prime}\right), \eta\left(t^{\prime}\right)\right)=\int_{0}^{t} \mathrm{~d} t^{\prime}(i \hat{\eta} \dot{\eta}+w i \hat{\eta} \eta)
$$

where the notation $w=\mu-\lambda$ has been introduced, and the interaction $S_{1}$ has the expression :

$$
S_{1}=\int_{0}^{t} \mathrm{~d} t^{\prime} \mathfrak{H}_{1}\left(i \hat{\eta}\left(t^{\prime}\right), \eta\left(t^{\prime}\right)\right)=\int_{0}^{t} \mathrm{~d} t^{\prime}\left[-\lambda(i \hat{\eta})^{2} \eta+v i \hat{\eta}(i \hat{\eta}+1) \eta^{2}\right]
$$

Since $S_{0}$ is quadratic in $\eta, \hat{\eta}$ the functional integral of $\exp \left(-S_{0}\right)$ can be evaluated exactly. Perturbation theory is then obtained by expanding $\exp \left(-S_{1}\right)$ as follows :

$$
\exp \left(-S_{1}\right)=\sum_{n=0}^{\infty} \frac{(-1)^{n}}{n !}\left(\int_{0}^{t} \mathrm{~d} t^{\prime} \mathscr{H}_{1}\left(i \hat{\eta}\left(t^{\prime}\right), \eta\left(t^{\prime}\right)\right)\right)^{n}=\sum_{n=0}^{\infty}(-1)^{n} \int_{0}^{t} \mathrm{~d} t_{n} \int_{0}^{t_{n}} \mathrm{~d} t_{n-1} \cdots \int_{0}^{t_{2}} \mathrm{~d} t_{1} \prod_{l=1}^{n} \mathscr{H}_{1}\left(i \hat{\eta}\left(t_{l}\right), \eta\left(t_{l}\right)\right) .
$$

The problem reduces to the evaluation of integrals of the form

$$
I_{n m}\left(t_{l+1}, t_{l}\right)=\int \mathfrak{D} \eta \mathfrak{D} \hat{\eta} \eta^{n}\left(t_{l+1}\right)\left(i \hat{\eta}\left(t_{l}\right)\right)^{m} \exp \left\{-\int_{t_{l}}^{t_{l+1}} \mathrm{~d} t^{\prime} \mathcal{H}_{0}\left(i \hat{\eta}\left(t^{\prime}\right), \eta\left(t^{\prime}\right)\right)\right\}
$$

Since $\mathfrak{H}_{0}$ is a quadratic form in the fields $i \hat{\eta}, \eta$, a form of Wick's theorem holds which allows one to reduce $I_{n m}$ to products of pairings of the form

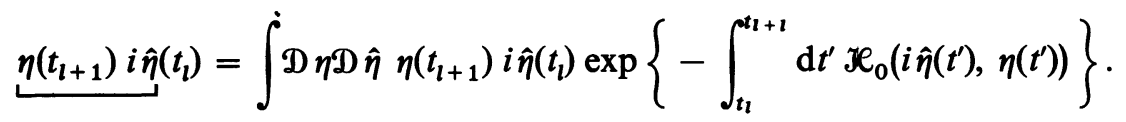

Explicit evaluation of the Gaussian integral allows one to express the pairing as the inverse, evaluated between $t_{l+1}$ and $t_{l}$, of the operator

$$
D\left(t-t^{\prime}\right)=\left[\frac{\mathrm{d}}{\mathrm{d} t}+w\right] \delta\left(t-t^{\prime}\right)
$$

The inverse is defined once the boundary condition is given. A look at the discretized form (2.23) of the functional integral suggests to supplement (3.26) with the boundary condition

$$
D^{-1}\left(t-t^{\prime}\right)=0, \quad t \leqslant t^{\prime}
$$

We obtain therefore :

$$
\eta(t) i \hat{\eta}\left(t^{\prime}\right)=\theta\left(t-t^{\prime}\right) \exp \left[-w\left(t-t^{\prime}\right)\right] .
$$

One can thus derive diagrammatic rules for the perturbative evaluation of any given correlation function. To be specific, let us consider the average number of particles $\langle n(t)\rangle$ at time $t$ in a state in which, at $t=0$, there was just one particle. The quantity to be computed is

$$
\langle n(t)\rangle=\left\langle\left|\pi a U_{t} \pi\right| 0\right\rangle,
$$

which corresponds to the following functional inte- 
gral :

$$
\begin{aligned}
\langle n(t)\rangle=\int \frac{\mathrm{d} \eta \mathrm{d} \eta^{\prime}}{2 \pi} \frac{\mathrm{d} \eta_{0} \mathrm{~d} \eta_{0}^{\prime}}{2 \pi} \mathrm{e}^{\eta-i \eta \eta^{\prime}} \eta U_{t}\left(i \eta^{\prime}, \eta_{0}\right) \mathrm{e}^{-i \eta \eta_{0} o^{\prime}} i \eta_{0}^{\prime}= \\
\quad=\int D D \eta D \hat{\eta} \eta(t) \exp \{-S[i \hat{\eta}, \eta]\}(i \hat{\eta}(0)+1) .
\end{aligned}
$$

Since $\langle|a| 0\rangle=0$ we may dispose of the one in the last factor. When we apply the expansion (3.23) to this expression we end up obtaining a network of pairings which may be conveniently expressed by graphs. Things may be simplified by considering that the identity

$$
\underbrace{\eta\left(t_{1}\right) i \hat{\eta}}\left(t_{0}\right)=\underbrace{\eta\left(t_{1}\right) i \hat{\eta}}\left(t^{\prime}\right) \underbrace{\eta\left(t^{\prime}\right) i \hat{\eta}}\left(t_{0}\right), \quad t_{0}<t^{\prime}<t_{1},
$$

allows one to consider just pairings among successive time arguments. If we now go to the Laplace transform of $\langle n(t)\rangle$ and take into account that

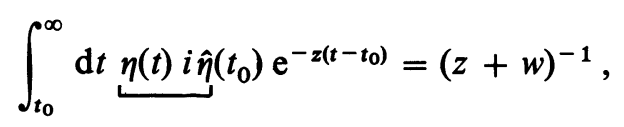

we obtain the following rules.

To compute $\langle n(t)\rangle$ to $m$-th order in $\mathfrak{H}_{1}$ :

(i) Draw $m$ vertices corresponding to monomials appearing in $\mathfrak{H}_{1}$ ordered in time from left to right in all possible distinct ways. To a vertex of the form $g_{k l}(i \hat{\eta})^{k} \eta^{l}$ attach $l$ « stumps » coming from the left (earlier times) and $k$ « stumps " going to the right (later times).

(ii) Draw a stump going to the right at the left of all vertices (it corresponds to $i \hat{\eta}(0)$ ) and one coming from the left at the right of all vertices (it corresponds to $\eta(t))$.

(iii) Join with a line each stump going to the right to one coming from the left at any later vertex, in all possible ways.

(iv) The contribution of any such time-ordered diagram is given by : (a) a product of $(m+1)$ dynamical factors, each given by $\left(z+N_{l} w\right)^{-1}$, where $N_{l}$ is the number of lines crossing the $l$-th time interval (from $t_{l-1}$ to $t_{l}$ ); (b) a product of $m$ vertex contributions, each given by $\left(-g_{k l}\right)$ where $g_{k l}$ is the coefficient of $(\mathrm{i} \hat{\eta})^{k} \eta^{l}$ in $\mathcal{H}_{1}$, as given above; (c) a symmetry factor, given by $1 / l$ ! for each group of $l$ lines connecting the same two vertices.

Let us remark that all diagrams appearing in this procedure are necessarily connected to the « external stumps » (rule (ii)). Moreover, all lines connect a vertex at earlier times to one at later times, so that causality is automatically satisfied. This is a consequence of the discretized form (2.23). The rules given above reduce, for the case of Schlogl's first model at hand, to the perturbative rules of Reggeon Quantum Mechanics which have been investigated by Ciafaloni and Onofri [35]. They are strongly related to the rules of time-ordered diagrams in the theory of dynamic critical phenomena, as given for instance by Halperin,
Hohenberg and Siggia [36], and by $\mathrm{Ma}$ [37]. It is obvious how they are generalized to the case where several species of particles are present.

\section{Birth-death processes on a lattice.}

Most aggregation processes we are interested in can be defined as birth-death processes, what makes the formalism described in the previous section suitable for their treatment. We shall now discuss the most basic process, namely the diffusion of independent particles. We shall then treat some simple aggregation processes without memory.

The microscopic states of birth-death processes on a lattice are identified by giving the occupation number $\underline{n}=\left\{n_{r}\right\}$ for each point $r$ of the lattice. One introduces therefore the base states $|\underline{n}\rangle$ and straightforwardly defines the Hilbert scalar product :

$$
\langle\underline{m} \mid \underline{n}\rangle=\prod_{r} m_{r} ! \delta_{m_{r}, n_{r}} .
$$

To each point $r$ of the lattice one associates a pair $\left(a_{r}, \pi_{r}\right)$ of annihilation and creation operators, satisfying the commutation relation :

$$
\left[a_{r}, \pi_{r^{\prime}}\right]=\delta_{r r^{\prime}} .
$$

Their action on the base states $\left|\ldots, n_{r}, \ldots\right\rangle$ is given by :

$$
\begin{gathered}
a_{r}\left|\ldots, n_{r}, \ldots\right\rangle=n_{r}\left|\ldots, n_{r}-1, \ldots\right\rangle, \\
\pi_{r}\left|\ldots, n_{r}, \ldots\right\rangle=\left|\ldots, n_{r}+1, \ldots\right\rangle .
\end{gathered}
$$

The macroscopic state $|\phi\rangle$ corresponding to the probabilities $\phi(n)$ of finding the system in the state $|\underline{n}\rangle$ is given by :

$$
|\phi\rangle=\sum_{\underline{n}} \phi(\underline{n})|\underline{n}\rangle .
$$

The corresponding generating function is given by :

$$
\phi(z)=\sum_{\underline{n}} \prod_{r} z_{r}^{n_{r}} \phi(\underline{n}) .
$$


The process we shall first consider is particle diffusion. One considers particles on the sites of the lattice which may jump independently, with a rate $w$, to any nearest neighbour of the site they are on. We have therefore the following master equation :

$$
\frac{\partial}{\partial t} \phi(\underline{n} ; t)=\sum_{r} \sum_{e} w\left[\left(n_{r}+1\right) \phi\left(\ldots, n_{r+e}-1, n_{r}+1, \ldots ; t\right)-n_{r} \phi\left(\ldots, n_{r+l, e}, n_{r}, \ldots ; t\right)\right] .
$$

The first sum runs over all sites of the lattice, and the second sum runs over the vectors $e$ which join any site to each of its nearest neighbours (nearest neighbour vectors).

The Liouvillian has therefore the following expression :

$$
L=\sum_{r} \sum_{e} w\left(\pi_{r+e} a_{r}-\pi_{r} a_{r}\right) .
$$

By applying the procedures sketched in the previous section it is easy to obtain the following functional integral expression for the kernel of the evolution operator $U_{t}=\exp (t L)$ :

$$
\begin{gathered}
U_{t}(\underline{z}, \underline{\zeta})=\int \prod_{r} \mathfrak{D} \eta_{r}\left(t^{\prime}\right) \mathfrak{D} \eta_{r}^{\prime}\left(t^{\prime}\right) \\
\exp \left\{-\int_{0}^{t} \mathrm{~d} t^{\prime}\left[\sum_{r} i \eta_{r}^{\prime} \dot{\eta}_{r}-\sum_{r} \sum_{e} w\left(i \eta_{r+e}^{\prime} \eta_{r}-i \eta_{r}^{\prime} \eta_{r}\right)\right]+\sum_{r} i \eta_{r}^{\prime}(t) \eta_{r}(t)\right\} .
\end{gathered}
$$

The expression is square brackets may be written, by means of a summation by parts, in the following form :

$$
\begin{array}{r}
\mathscr{H}=\sum_{r} i \eta_{r}^{\prime} \dot{\eta}_{r}-\sum_{r} w i \eta_{r}^{\prime}\left[\sum_{e}\left(\eta_{r+e}-\eta_{r}\right)\right]= \\
=\sum_{r} i \eta_{r}^{\prime}\left(\dot{\eta}_{r}-w \Delta \eta_{r}\right),
\end{array}
$$

where $\Delta$ is the discrete Laplacian, which for a lattice with coordination number $q$ is given by :

$$
\Delta \eta_{r}=\sum_{e} \eta_{r+e}-q n_{r} .
$$

Equation (4.9) must be supplemented by the boundary conditions :

$$
i \eta_{r}^{\prime}(t)=z_{r} ; \quad \eta_{r}(0)=\zeta_{r} ; \quad \forall r .
$$

The functional integral appearing in equation (4.9) may be evaluated exactly. The integration over $\eta^{\prime}$ yields as a constraint the diffusion equation which must be satisfied by $\underline{\eta}(t)$ :

$$
\frac{\mathrm{d} \eta_{r}(t)}{\mathrm{d} t}=w \Delta \eta_{r}(t)
$$

Let $H(t, \eta)$ the solution of this equation which satisfies the initial condition :

$$
H_{r}(0, \underline{\eta})=\eta_{r} .
$$

The kernel $U_{t}(z, \underline{\zeta})$ then has the following expression :

$$
U_{t}(\underline{z}, \underline{\zeta})=\exp \left[\sum_{r} z_{r} H_{r}(t, \underline{\zeta})\right]
$$

The average occupation number at site $r$ is given by :

$$
v_{r}(t)=\left\langle n_{r}(t)\right\rangle=\int \prod_{r^{\prime}}\left(\frac{\mathrm{d} z_{r^{\prime}} \mathrm{d} z_{r^{\prime}}^{\prime}}{2 \pi} \frac{\mathrm{d} \zeta_{r^{\prime}} \mathrm{d} \zeta_{r^{\prime}}^{\prime}}{2 \pi}\right) \exp \left(\sum_{r^{\prime}} z_{r^{\prime}}\right) z_{r} U_{t}\left(i \underline{z}^{\prime}, \underline{\zeta}\right) \exp \left(-i \sum_{r^{\prime}} \zeta_{r^{\prime}} \zeta_{r^{\prime}}^{\prime}\right) \Phi_{0}\left(i \underline{\zeta}^{\prime}\right),
$$

where $\Phi_{0}(\zeta)$ is the generating function of the initial macroscopic state. By substituting equation (4.15) and performing the integrals over $z, z^{\prime}$ we obtain

$$
v_{r}(t)=\int \prod_{r^{\prime}}\left(\frac{\mathrm{d} \zeta_{r^{\prime}} \mathrm{d} \zeta_{r^{\prime}}^{\prime}}{2 \pi}\right) H_{r}(t, \underline{\zeta}) \exp \left(-i \sum_{r^{\prime}} \zeta_{r^{\prime}} \zeta_{r^{\prime}}^{\prime}\right) \Phi_{0}\left(i z^{\prime}\right)
$$

It is easy to check, via the definition of $H(t, \zeta)$, that $\underline{v}(t)$ does satisfy the diffusion equation (Eq. (4.13)). $\overline{\mathrm{A}}$ special case of interest is when $\Phi_{0}$ corresponds to a probability distribution which is an independent Poisson distribution for each lattice site, with average $\underline{v}^{0} ;$ then

$$
\Phi_{0}(\underline{\zeta})=\exp \left[\sum_{r} v_{r}^{0}\left(\zeta_{r}-1\right)\right] .
$$

One checks that the generating function at time $t$ is 
given by :

$$
\begin{aligned}
\Phi_{t}(\underline{\zeta})= & \int \prod_{r}\left(\frac{\mathrm{d} z_{r} \mathrm{~d} z_{r}^{\prime}}{2 \pi}\right) \times \\
& \times \exp \left\{\sum_{r}\left[\zeta_{r} H_{r}(t, \underline{z})-i z_{r} z_{r}^{\prime}+v_{r}^{0}\left(i z_{r}^{\prime}-1\right)\right]\right\} .
\end{aligned}
$$

One has therefore

$$
\Phi_{t}(\underline{\zeta})=\exp \left[\sum_{r} \zeta_{r} H_{r}\left(t, \underline{v}^{0}\right)-v_{r}^{0}\right]
$$

which, since the particle number is conserved :

$$
\sum_{r} v_{r}^{0}=\sum_{r} H_{r}\left(t, v^{0}\right)
$$

may be written as follows :

$$
\Phi_{t}(\underline{\zeta})=\exp \left[\sum_{r} H_{r}\left(t, \underline{v}^{0}\right)\left(\zeta_{r}-1\right)\right],
$$

corresponding to a Poisson distribution with average $\underline{H}\left(t, v^{0}\right)$.

The simplest generalization of this process corresponds to the case in which the jump rate $w$ depends on the site $r$, the jump direction $e$ and possibly on time $t$. This is the case of biased diffusion, which is relevant to the discussion of random walks with memory. The master equation has the form :

$$
\frac{\partial|\phi\rangle}{\partial t}=\sum_{r} \sum_{e} w_{r, e}(t)\left[\pi_{r+e} a_{r}-\pi_{r} a_{r}\right]|\phi\rangle .
$$

By straightforward manipulations it is possible to introduce a local diffusion $w_{r}(t)$, equal to the average of the $w_{r, e}(t)$ with respect to $e$, and a local drift $b_{r, e}(t)$, and write the Liouvillian of this equation in the following form :

$$
\begin{aligned}
L=\sum_{r} \sum_{e} w_{r}(t)\left(\pi_{r+e}-\pi_{r}\right) a_{r}+ \\
\quad+\sum_{r} \sum_{e} b_{r, e}(t)\left(\pi_{r+e}-\pi_{r}\right) a_{r} .
\end{aligned}
$$

The action of biased diffusion is therefore given by :

$$
\begin{aligned}
S=\int_{0}^{t} \mathrm{~d} t^{\prime} & \left\{\sum_{r} i \eta_{r}^{\prime} \dot{\eta}_{r}-\sum_{r} w_{r}\left(t^{\prime}\right) \Delta\left(i \eta_{r}^{\prime}\right) \eta_{r}-\right. \\
& \left.-\sum_{r}\left[\sum_{e} b_{r, e}\left(i \eta_{r+e}^{\prime}-i \eta_{r}^{\prime}\right) \eta_{r}\right]\right\} .
\end{aligned}
$$

In the continuum limit, if $w_{r}(t)$ is uniform in space and constant in time, we recover the action for biased diffusion proposed e.g. in reference [16]. One only has to make the correspondences

$$
\begin{aligned}
i \hat{\eta} \rightarrow \hat{\psi} ; \eta \rightarrow & \psi ; a_{0}^{2} w \rightarrow \mathfrak{D} ; \\
& 2 a_{0}\left(b_{r, e}-b_{r,-e}\right) \rightarrow \underline{b} . \hat{\mathbf{e}},
\end{aligned}
$$

where $a_{0}$ is the lattice constant and $\hat{\mathbf{e}}$ is the unit vector in direction $e$, to obtain equations (13), (14) of that reference.

The case of random walks with traps is also easily treated. It belongs to the simplest class of birth-death processes on a lattice, to which we now turn our attention. We consider a process in which each particle at site $r$ faces the following possibilities : either it may jump (with rate $w$ ) to one of the nearest neighbours of the site, or it may give origin with rate $\lambda_{k}$ to $k$ particles still located at site $r$. The rate $\lambda_{k}$ may only depend on the occupation number of site $r$. It may be therefore expressed in terms of the factorial products $(n)_{m}$ defined by

$$
(n)_{m}=n(n-1) \ldots(n-m+1) .
$$

Let $\lambda_{k}(n)$ be given by

$$
\lambda_{k}(n)=\sum_{m} \lambda_{k m}(n)_{m} .
$$

The master equation of this process is given by :

$$
\begin{aligned}
\frac{\partial}{\partial t} \phi(\underline{n}, t) & =\sum_{r} \sum_{e} w\left[\left(n_{r}+1\right) \phi\left(\ldots, n_{r+e}-1, n_{r}+1, \ldots ; t\right)-n_{r} \phi\left(\ldots, n_{r+e}, n_{r}, \ldots ; t\right)\right] \\
& +\sum_{r} \sum_{k}\left[\left(n_{r}-k+1\right) \lambda_{k}\left(n_{r}-k+1\right) \phi\left(\ldots, n_{r}-k+1, \ldots ; t\right)-n_{r} \lambda_{k}\left(n_{r}\right) \phi\left(\ldots, n_{r}, \ldots ; t\right)\right] .
\end{aligned}
$$

The Liouvillian has therefore the expression

$$
L=\sum_{r} \sum_{e} w\left(\pi_{r+e}-\pi_{r}\right) a_{r}+\sum_{r} \sum_{k m} \lambda_{k m}\left[\pi_{r}^{k+m+1}-\pi_{r}^{m+1}\right] a_{r}^{m+1} .
$$

The kernel of the evolution operator has therefore the expression

$$
\begin{aligned}
U_{t}(\underline{z}, \underline{\zeta})=\int \mathfrak{D} \underline{\eta} \mathfrak{D} \underline{\eta^{\prime}} \exp \{ & -\int_{0}^{t} \mathrm{~d} t^{\prime}\left[\sum_{r} i \eta_{r}^{\prime} \dot{\eta}_{r}-\sum_{r} w \Delta\left(i \eta_{r}^{\prime}\right) \eta_{r}-\right. \\
& \left.\left.-\sum_{r} \sum_{k m} \lambda_{k m}\left[\left(i \eta_{r}^{\prime}\right)^{k}-1\right]\left(i \eta_{r}^{\prime}\right)^{m+1} \eta_{r}^{m+1}\right]+\sum_{r} z_{r} \eta_{r}(t)\right\} .
\end{aligned}
$$


It is convenient to perform the shift

$$
i \underline{\eta}^{\prime} \rightarrow i \underline{\hat{\eta}}=i \underline{\eta}^{\prime}-1,
$$

and to rearrange the terms of equation (4.30) to obtain the action

with

$$
S=S_{0}+S_{1},
$$

$S_{0}=\int_{0}^{t} \mathrm{~d} t^{\prime}\left[\sum_{r} i \hat{\eta}_{r} \dot{\eta}_{r}-\sum_{r} w \Delta\left(i \hat{\eta}_{r}\right) \eta_{r}-\sum_{r} \lambda i \hat{\eta}_{r} \eta_{r}\right]$,

where

and

$$
\lambda=\lambda_{00}
$$

$$
S_{1}=\int_{0}^{t} \mathrm{~d} t^{\prime}\left[\sum_{r} \sum_{m n} g_{m n}\left(i \hat{\eta}_{r}\right)^{m} \eta_{r}^{n}\right]
$$

where the $g_{m n}$ can be expressed in terms of the $\lambda_{k m}$. In this way no terms linear in $\eta$ appear in $S_{1}$. Attaching a site label to each vertex in perturbation theory and associating to the line going from $r$ to $r^{\prime}$ the pairing

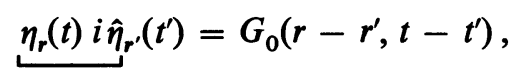

where $G_{0}\left(r-r^{\prime}, t-t^{\prime}\right)$ is the solution of the equation

$$
\left[\frac{\partial}{\partial t}-\lambda-w \Delta\right] G_{0}\left(r-r^{\prime}, t-t^{\prime}\right)=\delta_{r r^{\prime}} \delta\left(t-t^{\prime}\right)
$$

satisfying the boundary condition

$$
G_{0}\left(r-r^{\prime}, t-t^{\prime}\right)=0, \quad t \leqslant t^{\prime},
$$

one recovers the perturbation theory for these processes in a standard way. Associating a wavevector $q$ to each line in a graph, taking into account wavevector conservation at each vertex, one associates with each time interval in time-ordered graphs (as defined in the previous sections) the contribution

$$
\left[z+N_{l} \lambda+\sum_{j} \varepsilon\left(\mathbf{q}_{j}\right)\right]^{-1}
$$

where

$$
\varepsilon(q)=w \sum_{e}[\exp (i q . e)-1]
$$

and the sum over $j$ runs over the $N_{l}$ lines belonging to the $l$-th time interval.

In the case of random walk with traps, whose density $\rho$ may depend on the site $r$ and on the time label $t$, we have

$$
\lambda_{00}=g \rho_{r}(t),
$$

and we may write

$$
\begin{aligned}
S & =S_{0}= \\
& =\int_{0}^{t} \mathrm{~d} t^{\prime}\left[\sum_{r} i \hat{\eta}_{r} \dot{\eta}_{r}-\sum_{r} w \Delta\left(i \hat{\eta}_{r}\right) \eta_{r}-\sum_{r} g i \hat{\eta}_{r} \rho_{r}(t) \eta_{r}\right],
\end{aligned}
$$

which is equivalent to equations (4), (5) of reference [13] in the continuum limit. (Please note that the sign of equation (4), this reference, is wrong.) In the case in which the local birth-death process corresponds to Schlögl's first model, namely when

$$
\lambda_{k}(n)=\lambda \delta_{k, 2}+(\mu+v(n-1)) \delta_{k, 0},
$$

we have the same « free " action as in equation (4.33), and the interaction

$$
S_{1}=\int_{0}^{t} \mathrm{~d} t^{\prime}\left[\sum_{r} \mathscr{X}_{1}\left(i \hat{\eta}_{r}\left(t^{\prime}\right), \eta_{r}\left(t^{\prime}\right)\right)\right]
$$

where $\mathscr{H}_{1}$ is the same interaction Lagrangian given for a single site in equation (3.22). We obtain therefore the action of Reggeon Field Theory [17-20].

\section{Birth-death processes with memory.}

We wish to describe in this section how the formalism of the previous sections can be applied to justify the field theories of irreversible processes which have been recently proposed, e.g. in references $[12,13,22]$. The main device is a suggestion by Grassberger [31] and Cardy and Grassberger [38] (see also Janssen [39]) to transform the non-Markovian evolution equation of a birth-death process with memory into a Markovian one by introducing some suitable "bookkeeping " variables.

To be definite let us consider the following kinetic version of the self-avoiding walk (SAW). We consider a walker which undergoes (unbiased) diffusion but is suppressed (with a small probability per unit time) whenever it steps on a lattice site which he has previously visited. In order to take account of this, we introduce, besides the walker's occupation number relative to site $r, m_{r}$, a corresponding bookkeeping variable $n_{r}$ which increases by one each time the walker leaves the lattice site. The walker then undergoes a random walk with trap density proportional to $n_{r}(t)$. We then have the following master equation :

$$
\begin{aligned}
\frac{\partial}{\partial t} \Phi\left(\ldots, m_{r}, n_{r}, \ldots ; t\right)=\sum_{r} \sum_{e} w[ & \left(m_{r}+1\right) \Phi\left(\ldots, m_{r+e}-1, n_{r+e}-1, m_{r}+1, n_{r}, \ldots ; t\right)- \\
& \left.-m_{r} \Phi\left(\ldots, m_{r+e}, n_{r+e}, m_{r}, n_{r}, \ldots ; t\right)\right] \\
& +\sum_{r} \tau\left[\left(m_{r}+1\right) n_{r} \Phi\left(\ldots, m_{r}+1, n_{r}, \ldots ; t\right)-m_{r} n_{r} \Phi\left(\ldots, m_{r}, n_{r}, \ldots ; t\right)\right] .
\end{aligned}
$$


We can now go to the operator representation of this evolution equation, by introducing the creation and annihilation operators for the walker, $\psi_{r}^{+}, \psi_{r}$, and the corresponding ones for the traps, $\phi_{r}^{+}, \phi_{r}$. We obtain therefore the following Liouvillian :

$$
L=\sum_{r} \sum_{e} w\left(\phi_{r}^{+} \psi_{r+e}^{+} \psi_{r}-\psi_{r}^{+} \psi_{r}\right)+\sum_{r} \tau\left(\phi_{r}^{+} \phi_{r} \psi_{r}-\phi_{r}^{+} \psi_{r}^{+} \phi_{r} \psi_{r}\right) .
$$

One obtains straightforwardly the following action for the path integral formulation of this process :

$$
S=\int_{0}^{t} \mathrm{~d} t^{\prime}\left[\sum_{r}\left(i \psi_{r}^{\prime} \dot{\psi}_{r}+i \phi_{r}^{\prime} \dot{\phi}_{r}\right)-\sum_{r} \sum_{e} w\left(i \phi_{r}^{\prime} i \psi_{r+e}^{\prime} \psi_{r}-i \psi_{r}^{\prime} \psi_{r}\right)-\sum_{r} \tau\left(i \phi_{r}^{\prime} \phi_{r} \psi_{r}-i \phi_{r}^{\prime} i \psi_{r}^{\prime} \phi_{r} \psi_{r}\right)\right] .
$$

It is convenient, also in this case, to perform the shift :

$$
i \underline{\phi^{\prime}} \rightarrow i \underline{\phi}=i \underline{\phi}^{\prime}-1 ; \quad i \underline{\psi^{\prime}} \rightarrow i \underline{\psi}=i \underline{\psi^{\prime}}-1 .
$$

We obtain therefore the following action :

$$
S=\int_{0}^{t} \mathrm{~d} t^{\prime}\left[\mathscr{H}_{0}+\mathscr{H}_{1}+\mathscr{H}_{2}\right]
$$

where the Lagrangians $\mathcal{H}$ are given by :

$$
\begin{aligned}
& \mathscr{H}_{0}=\sum_{r}\left(i \hat{\psi}_{r} \dot{\psi}_{r}-w i \hat{\psi}_{r} \Delta \psi_{r}+i \hat{\phi}_{r} \dot{\phi}_{r}\right), \\
& \mathscr{H}_{1}=\sum_{r}\left[\left(\sum_{e} w i \hat{\phi}_{r} i \hat{\psi}_{r+e} \psi_{r}\right)-\tau i \hat{\psi}_{r} \phi_{r} \psi_{r}\right], \\
& \mathscr{H}_{2}=\sum_{r}\left(-q w i \hat{\phi}_{r} \psi_{r}+\tau i \hat{\phi}_{r} i \hat{\psi}_{r} \phi_{r} \psi_{r}\right) .
\end{aligned}
$$

We have denoted by $q$ the coordination number of the lattice. One needs not to take into account the third term, $\mathrm{Je}_{2}$ if one is only interested in the asymptotic properties of the walk. Its first term, indeed, cannot contribute to quantities such as the probability $G\left(r, t ; r_{0}, t_{0}\right)$ that a walk originated at $r_{0}$ at time $t_{0}$ ends up at $r$ at time $t$ (it must be taken into account in the many walker problem). The second term is higher order in the fields and therefore irrelevant. Let us thus neglect $\mathfrak{H}_{2}$ for the moment. One can then integrate out the bookkeeping fields $i \hat{\phi}, \phi$ by means of the relation

$$
\begin{array}{r}
\int \mathfrak{D} \underline{\hat{\phi}} \mathfrak{D} \underline{\exp }\left[-\int_{0}^{t} \mathrm{~d} t^{\prime} \sum_{r}\left(i \hat{\phi}_{r} \dot{\phi}_{r}+\lambda_{r} i \hat{\phi}_{r}+\mu_{r} \phi_{r}\right)\right]=\int \mathfrak{D} \underline{\exp }\left(-\int_{0}^{t} \mathrm{~d} t^{\prime} \sum_{r} \mu_{r} \phi_{r}\right) \delta\left[\dot{\phi}_{r}+\lambda_{r}\right]= \\
=\exp \left[\int_{0}^{t} \mathrm{~d} t^{\prime} \sum_{r} \mu_{r} \int_{0}^{t^{\prime}} \mathrm{d} t^{\prime \prime} \lambda_{r}\left(t^{\prime \prime}\right)\right] .
\end{array}
$$

We have thus a Lagrangian which only involves $\underline{\hat{\psi}}, \underline{\psi}$ :

$$
\begin{aligned}
& \mathscr{H}_{0}^{\prime}=\sum_{r}\left(i \hat{\psi}_{r} \dot{\psi}_{r}-w i \hat{\psi}_{r} \Delta \psi_{r}\right), \\
& \mathcal{H}_{1}^{\prime}=\sum_{r} \tau i \hat{\psi}_{r} \psi_{r} \int_{0}^{t} \mathrm{~d} t^{\prime}\left(\sum_{e} w i \hat{\psi}_{r+e}\left(t^{\prime}\right) \psi_{r}\left(t^{\prime}\right)\right) .
\end{aligned}
$$

One should compare this expression with the Lagrangian conjectured for the same problem in reference [13]. In the continuum limit - and neglecting irrelevant terms - the two coincide up to the identification

$$
\tau q w \rightarrow g_{1} \text {. }
$$

One can extend the same reasoning to obtain a path integral description of several other models of irre- versible behaviour. Let us consider e.g. the " true " self-avoiding walk (TSAW) [9]. In this case the walker undergoes biased diffusion with a bias which is a function of the number $n_{r}$ of times the site $r$ has been visited in the past :

$$
w_{r, e}=w q \mathrm{e}^{-g n_{r+e}} / \sum_{e^{\prime}} \mathrm{e}^{-g n_{r+e^{\prime}}} .
$$

The sum runs over the $q$ nearest neighbours of the site $r$. In the limit of weak interaction, $g \ll 1$, we have

$$
w_{r, e}=w\left[1-g\left(n_{r+e}-\frac{1}{q} \sum_{e^{\prime}} n_{r+e^{\prime}}\right)\right] .
$$

One can introduce a pair of bookkeeping operators, $\phi_{r}^{+}, \phi_{r}$, for each lattice site, besides the creation and 
annihilation operators for the walker, $\psi_{r}^{+}, \psi_{r}$. One can thus obtain the Liouvillian

$$
L=\sum_{r} \sum_{e} w\left[1-g\left(\phi_{r+e}^{+} \phi_{r+e}-\frac{1}{q} \sum_{e^{\prime}} \phi_{r+e^{\prime}}^{+} \phi_{r+e^{\prime}}\right)\right]\left(\phi_{r}^{+} \psi_{r+e}^{+} \psi_{r}-\psi_{r}^{+} \psi_{r}\right) .
$$

Going to the path integral representation and performing the usual shift we obtain the action :

$$
\begin{array}{r}
S=\int_{0}^{t} \mathrm{~d} t^{\prime}\left[\sum_{r}\left(i \hat{\psi}_{r} \dot{\psi}_{r}+i \hat{\phi}_{r} \dot{\phi}_{r}\right)-\sum_{r} \sum_{e} w\left\{1-g\left[i \hat{\phi}_{r+e} \phi_{r+e}-\frac{1}{q} \sum_{e^{\prime}} i \hat{\phi}_{r+e^{\prime}} \phi_{r+e^{\prime}}+\phi_{r+e}-\frac{1}{q} \sum_{e^{\prime}} \phi_{r+e^{\prime}}\right]\right\} \times\right. \\
\left.\left[\left(i \hat{\psi}_{r+e}-i \hat{\psi}_{r}\right) \psi_{r}+i \hat{\phi}_{r} \psi_{r}+i \hat{\phi}_{r} i \hat{\psi}_{r+e} \psi_{r}\right]\right]
\end{array}
$$

In the continuum limit, the factor in curly brackets may be approximated by :

$$
1-g e . \nabla \phi_{r}
$$

up to irrelevant terms; and neglecting likewise other irrelevant terms which arise in the product, we obtain the action

$$
\begin{aligned}
& S=\int_{0}^{t} \mathrm{~d} t^{\prime}\left\{\sum_{r}\left(i \hat{\psi}_{r} \dot{\psi}_{r}-w i \hat{\psi}_{r} \Delta \psi_{r}+i \hat{\phi}_{r} \dot{\phi}_{r}\right)+\right. \\
& \left.+\sum_{r}\left[g w\left(\nabla \phi_{r} \cdot \nabla\left(i \hat{\psi}_{r}\right)\right)-q w i \hat{\phi}_{r} i \hat{\psi}_{r} \psi_{r}+i \hat{\phi}_{r} \psi_{r}\right]\right\} .
\end{aligned}
$$

The last terms does not contribute to the probability $G\left(r, t ; r_{0}, t_{0}\right)$; and in the same way as before the rest may be shown to be equivalent to the Lagrangian of TSAW conjectured in reference [12].

The same sort of approach can be applied to obtain the field theoretical formulation of some irreversible aggregation models. I refer to $[38,39]$ for a treatment along these lines of a model introduced by Cardy [22] to represent epidemic processes with immunization. I only wish to point out that the same model, but with sensibilization in place of immunization, should belong to the same universality class as the Eden model [40]. This model is defined as follows : sites are of two kinds, « healthy " and " ill ». With a certain probability per unit time each "ill " site may effect one of its neighbours, still remaining ill. As a consequence, the cluster of ill sites grows without limit. The problem is to understand the geometric nature of the growing cluster. Cardy's model considers three populations of sites : " healthy ", « ill ", and " healed » sites. Ill sites may turn neighbouring sites into ill ones, but they do not always remain ill; they may turn into healed ones. Sensibility of healed sites may be lower than for healthy ones (immunization), or may be higher (sensibilization). In this last case one may envisage the situation in which " healed " sites become ill almost at once, and one is led back to the Eden model [41]. Cardy and Grassberger [38] and Janssen [39] show that the immunization case produces the statistics of percolation clusters in the static limit. On the other hand Cardy [22] has shown that in the sensibilization case the trivial - diffusion-dominated - fixed point of the renormalization group equations is unstable in all dimensions. This implies that there is no higher critical dimension for the Eden model.

A rather popular aggregation process is the WittenSander model [1] of diffusion-limited aggregation (DLA). In its " penetrable " version it may be defined as follows. There are two kinds of particles : aggregated A and diffusing D. A steady flow of diffusing particles comes in from infinity. They undergo a normal random walk, only that they may turn into aggregated ones (and stop) whenever they occupy a site neighbouring one already occupied by an A particle. On the other hand, A particles do not move.

We shall show in the following how the problem can be represented in our formalism and how the meanfield treatment introduced by Witten and Sander [1] and discussed by Nauenberg and collaborators $[4,5]$ may be recovered.

Introducing the occupation numbers $n_{r}$ of A particles and $m_{r}$ of $\mathrm{D}$ particles we have the master equation :

$$
\begin{aligned}
\frac{\partial}{\partial t} \Phi\left(\ldots, m_{r}, n_{r}, \ldots ; t\right) & =\sum_{r} \sum_{e}\left\{w \left[\left(m_{r}+1\right) \Phi\left(\ldots, m_{r+e}-1, n_{r+e}, m_{r}+1, n_{r}, \ldots ; t\right)-\right.\right. \\
& \left.-m_{r} \Phi\left(\ldots, m_{r+e}, n_{r+e}, m_{r}, n_{r}, \ldots ; t\right)\right]+\lambda\left[n_{r+e}\left(m_{r}+1\right) \Phi\left(\ldots, m_{r+e}, n_{r+e}, m_{r}+1, n_{r}-1, \ldots ; t\right)\right. \\
& \left.\left.-n_{r+e} m_{r} \Phi\left(\ldots, m_{r+e}, n_{r+e}, m_{r}, n_{r}, \ldots ; t\right)\right]\right\} .
\end{aligned}
$$

We thus obtain the Liouvillian

$$
L=\sum_{r} \sum_{e}\left\{w\left[\left(D_{r+e}^{+}-D_{r}^{+}\right) D_{r}\right]+\lambda\left[A_{r}^{+} A_{r+e}^{+} D_{r} A_{r+e}-D_{r}^{+} A_{r+e}^{+} D_{r} A_{r+e}\right]\right\} .
$$


The corresponding action (after the shift) is given by :

$$
S=\int_{0}^{t} \mathrm{~d} t^{\prime}\left\{\sum_{r}\left[i \hat{D}_{r} \dot{D}_{r}+i \hat{A}_{r} \dot{A}_{r}-w i \hat{D}_{r} \Delta D_{r}\right]-\sum_{r} \sum_{e} \lambda\left(1+i \hat{A}_{r+e}\right)\left(i \hat{A}_{r}-i \hat{D}_{r}\right) D_{r} A_{r+e}\right\}
$$

The usual mean-field theory $[1,4,5]$ can be obtained if one neglects the terms proportional to $i \hat{A}_{r+e}$ in this expression. The resulting functional is then linear in the " hat " fields, which may be integrated away, yielding two coupled differential equations :

$$
\begin{gathered}
\dot{D}_{r}=w \Delta D_{r}-\lambda D_{r} \sum_{e} A_{r+e} \\
\dot{A}_{r}=\lambda D_{r} \sum_{e} A_{r+e}
\end{gathered}
$$

Equations (5.22)-(5.23) correspond to equations (1a)(1b) of reference [5].

It is then apparent that one would go beyond mean field theory by considering the term proportional to $i \hat{A}_{r+e}$ as a perturbation. This program will be taken up in a future publication.

\section{Conclusions.}

We have shown how the Fock space techniques for classical objects, introduced by Doi [23] and reformulated by Grassberger and Scheunert [25], naturally leads to a path integral formulation of several interesting birth-death processes. By means of a simple device, their scope may be extended to encompass some models describing irreversible aggregation process. Such a formulation has the advantage of easily allowing for an estimation of the validity of approximations, or for building up a systematic perturbation scheme, which may eventually lead to the application of renormalization group methods.

The purpose of this paper will be reached, if the awareness of the existence of these methods spreads a little among the community of physicists working on the theory of aggregation and gelation. We avoided charging the paper with applications of the theory to several models, in order to make transparent the almost mechanical easiness by which irreversible aggregation processes are led to a master equation, then to a path integral representation. It is worthwhile to remark how several approximate procedures (such as the mean-field-like formulation of DLA) can be obtained by means of straightforward manipulations.

The scope of these methods widely extends beyond the realm of aggregation processes. We wish to relate, in a future publication, how they help in understanding the relation between classical and quantum statistics in laser-like systems, as well as how they provide new points of view on the nature of quasi-deterministic approximations in chemical systems.
We think it sufficient to have drawn the attention on these methods and to have proven by a few examples their importance and flexibility.

\section{Acknowledgments.}

This work was sparked by a conversation that the author had with Prof. P. Grassberger at the CECAM Workshop on Kinetics of Aggregation and Gelation (Orsay, France, Sept. 1984). He therefore warmly thanks Prof. Grassberger for his illuminating suggestions and the organizers of the workshop for having given him the opportunity of such a meeting. He thanks C. Margolinas for hospitality as well as F. De Pasquale, L. Pietronero and P. Tombesi for suggestions and criticisms. He is also grateful to the referees for having pointed out several misprints in the manuscript.

Note added in proof. - After this paper had been accepted for publication, I have been made aware that a similar formalism, based upon the work of Doi, had also been introduced by N. Goldenfeld (J. Phys. A : Math. Gen. 17 (1984) 2807). It is my hope that an expository account of this formalism, such as the present work, will make such rediscoveries unnecessary for the future.

\section{Appendix.}

INCLUSIVE AND EXCLUSIVE SCALAR PRODUCTS. - The formalism dealt with in this paper is based upon the scalar product (2.3), to which Grassberger and Scheunert [25] have given the name " exclusive product ", since it makes two states orthogonal, whenever they differ in any occupation number. They find more convenient to introduce and exploit the « inclusive product" defined by

$$
\langle n \mid m\rangle_{\text {in }}=\sum_{k} \frac{1}{k !}(n)_{k}(m)_{k}
$$

where $(n)_{k},(m)_{k}$ are the factorial products (4.26) :

$$
(n)_{k}=n(n-1)(n-k+1)=n ! /(n-k) ! .
$$

Since it is hard to work directly with the product (A.1), we shall exploit the following relation between the 
inclusive and exclusive products of any two vectors $|\phi\rangle|\chi\rangle$ :

$$
\langle\phi \mid \chi\rangle_{\text {in }}=\left\langle\phi\left|\mathrm{e}^{\pi} \mathrm{e}^{a}\right| \chi\right\rangle,
$$

where the second scalar product is evaluated with the rule (2.3). We have in fact for any pair of integers $n, m$ :

$$
\begin{aligned}
\left\langle n\left|\mathrm{e}^{\pi} \mathrm{e}^{a}\right| m\right\rangle & =\sum_{k} \frac{1}{k !}\left\langle n\left|\mathrm{e}^{\pi} a^{k}\right| m\right\rangle= \\
& =\sum_{k, l} \frac{1}{k !} \cdot \frac{1}{l !}(m)_{k}\left\langle n\left|\pi^{l}\right| m-k\right\rangle \\
& =\sum_{k} \frac{1}{k !}(m)_{k}(n)_{k}=\langle n \mid m\rangle_{\text {in }} .
\end{aligned}
$$

In this formalism the creation operator $\pi$, defined by equation (2.5), is no more the Hermitian conjugate of the destruction operator $a$. One has indeed :

$$
a^{+}=\pi-1,
$$

where the Hermitian conjugate is taken with respect to the scalar product (A.1). Equation (A.5) is the fundamental relation of the inclusive scalar product, which makes its use slightly simpler than the exclusive one.

The path integral formation can be now developed with this new product if a decomposition of the identity analogous to equation (2.11) is introduced. Let us define indeed the Poisson states

$$
|z\rangle=\sum_{n=0}^{\infty} \frac{z^{n}}{n !} \mathrm{e}^{-z}|n\rangle=\mathrm{e}^{z(\pi-1)}|0\rangle
$$

which satisfy

$$
a|z\rangle=z|z\rangle
$$

One then has

$$
\langle z \mid n\rangle_{\mathrm{in}}=\sum_{k=0}^{n} \frac{(n)_{k}}{k !} z^{n-k}=(1+z)^{n} .
$$

One obtains therefore

$$
\begin{aligned}
& \int \frac{\mathrm{d} z \mathrm{~d} z^{\prime}}{2 \pi} \mathrm{e}^{-i z z^{\prime}}|z\rangle\left\langle i z^{\prime} \mid n\right\rangle_{\text {in }}= \\
& =\sum_{k=0}^{\infty} \int \frac{\mathrm{d} z \mathrm{~d} z^{\prime}}{2 \pi}|k\rangle \frac{z^{k}}{k !}\left(1+i z^{\prime}\right)^{n} \mathrm{e}^{-i z z^{\prime}-z} \\
& =\sum_{k=0}^{\infty} \int \frac{\mathrm{d} z \mathrm{~d} \hat{z}}{2 \pi}|k\rangle \frac{1}{k !} z^{k}(i \hat{z})^{n} \mathrm{e}^{-i z \hat{z}}=|n\rangle .
\end{aligned}
$$

One thus obtains the following decomposition of the identity :

$$
1=\int \frac{\mathrm{d} z \mathrm{~d} z^{\prime}}{2 \pi}|z\rangle\left\langle i z^{\prime}\right| \mathrm{e}^{-i z z^{\prime}},
$$

from which, along the lines of section 2, one obtains the whole path integral formalism. The relation (A.5) makes it unnecessary to perform the shift (3.4) and the analogous ones in the integration variables. A similar simplification occurs in the expectation values, which are represented, within the inclusive scalar product formalism, by

$$
\langle A\rangle_{\phi}=\langle 0|A| \phi\rangle,
$$

instead of equation (2.27). Expectation values of normal products of the form

$$
\left(a^{+}\right)^{k} a^{l}=(\pi-1)^{k} a^{l}
$$

vanish unless $k=0$ and are otherwise given by

$$
\left\langle a^{l}\right\rangle_{\phi}=\int \frac{\mathrm{d} z \mathrm{~d} \hat{z}}{2 \pi} z^{l} \phi\left(i z^{\prime}\right) .
$$

By the use of the inclusive product one directly obtains the final formula of the path integral formalism. Its use is however slightly less intuitive and its introduction relies on the exclusive scalar product (2.3).

A formalism based on complex integrals can also be introduced with similar arguments.

\section{References}

[1] Witten Jr, T. A. and Sander, L. M., Phys. Rev. Lett. 47 (1980) 1400 and Phys. Rev. B 27 (1983) 5686.

[2] Meakin P. and Witten Jr, T. A., Phys. Rev. A 28 (1983) 2985.

[3] Plischke, M. and RaCz, Z., Phys. Rev. Lett. 53 (1980) 415.

[4] Nauenberg, M., Phys. Rev. B 28 (1983) 449.

[5] NAUEnberg, M., Richter, R. and SANDER, L. M., B 28 (1983) 1649.

[6] Muthukumar, M., Phys. Rev. Lett. 50 (1983) 839.

[7] Oвuкнov, S. P., Kinetically Aggregated Clusters, preprint (1984).

[8] Vicsex, T. and Family, F., Phys. Rev. Lett. 52 (19,84) 1669.
[9] Amit, D. J. Parisi, G. and Peliti, L., Phys. Rev. B 27 (1983) 1635.

[10] Majid, I., Jan, N., Coniglio, A. and Stanley, H. E., Phys. Rev. Lett. 52 (1984) 1257.

[11] Lyklema, J. W. and Kremer, K., J. Phys. A 17 (1984) L-691.

[12] Obukhov, S. P. and Peliti, L., J. Phys. A 16 (1983) L-147.

[13] Peliti, L., J. Physique Lett. 45 (1984) L-925.

[14] Pietronero, L., Phys. Rev. B 27 (1983) 5887.

[15] Pietronero, L., Survival Probability for Kinetic SelfAvoiding Walks, preprint (1984).

[16] Peliti, L., Phys. Rep. 103 (1984) 225. 
[17] Grassberger, P. and Sundermeyer, K., Phys. Lett. 77B (1978) 220.

[18] Grassberger, P. and de la Torre, A., Ann. Phys. 122 (1979) 373.

[19] Cardy, J. L. and Sugar, R. L., J. Phys. A 13 (1980) 2423.

[20] JANSSen, H. K., Z. Phys. B 42 (1981) 151.

[21] Grassberger, P., Z. Phys. B 47 (1982) 365.

[22] Cardy, J. L., J. Phys. A 16 (1983) L-709.

[23] DoI, M., J. Phys. A 9 (1976) 1465, 1479.

[24] Rose, H. A., J. Stat. Phys. 20 (1979) 415.

[25] Grassberger, P. and Scheunert, M., Fortschr. Phys. 28 (1980) 547.

[26] Martin, P. C., Siggia, E. D. and Rose, H. A., Phys. Rev. A 8 (1978) 423.

[27] De Dominicis, C., J. Physique 37 (1976) 247.

[28] JANSSEN, H. K., Z. Phys. B 23 (1976) 377.

[29] Gardiner, C. W. and Chaturvedi, S., J. Stat. Phys. 17 (1977) 429, 18 (1978) 501.

[30] ITZYKson, C. and ZuBER, J. B., Quantum Field Theory (McGraw-Hill, New York) 1980, p. 435.

[31] Grassberger, P., unpublished (1984).
[32] Rozanov, Y., Processus Aléatoires (Mir, Moscow) 1975, p. 139.

[33] Gardiner, C. W., Handbook of Stochastic Methods (Springer, Heidelberg) 1983, p. 38.

[34] Schlögl, F., Z. Phys. 253 (1972) 147.

[35] Ciafaloni, M. and Onofri, E., Nucl. Phys. B 151 (1979) 118.

[36] Halperin, B. I., Hohenberg, P. C. and Siggia, E. D., Phys. Rev. B 13 (1976) 1299.

[37] MA, S. K., Modern Theory of Critical Phenomena (Benjamin, New York) 1976.

[38] Cardy, J. L. and Grassberger, P., J. Phys. A 18 (1985) L-267.

[39] JANsSEN, H. K., Z. Phys. B 58 (1985) 311.

[40] EDEn, M., in Proceedings of the Fourth Berkeley Symposium on Mathematical Statistics and Probability, edited by J. Neyman (University of California Press, Berkeley) 1981, Vol. IV, p. 233.

[41] After completion of this work, a preprint by Y. Shapir and Y. C. Zhang was received, in which a field theory for the Eden model along lines similar to the present ones is proposed. 